\title{
Authors' reply to the Letter to the Editor of Edwin C.W. Lim concerning "Nucleus pulposus deformation in response to lumbar spine lateral flexion: an in vivo MRI investigation", Eur Spine J (2010) 19:1115-1120 by Fazey PJ et al.
}

\author{
Peter J. Fazey
}

Received: 22 August 2010/Published online: 2 September 2010

(C) Springer-Verlag 2010

\section{Authors' response}

The methodology, as described, involved the placement of three lines across the disc orthogonal to the para-median sagittal axis; a central line followed by adjacent lines anterior and posterior with spacing standardized by the operator as depicted in Fig. 2.

The use of three lines represented an imposed limitation of the software routine to average derived data. This

method was not claimed to be optimal only more representative of the hydration profile than data from a single line sample as has been reported previously. Regardless of the number of lines used, the analysis is limited to two dimensions; three dimensional imaging and analysis of the nucleus pulposus would be optimal as noted in the original article. 